\title{
Estimation of the Oxidative Deterioration of Turbine Oil Using Membrane Patch Color
}

\author{
Tomohiko Kon, ${ }^{1}$ Tomomi Honda $\mathbb{D D}^{2}$ and Akira Sasaki ${ }^{3}$ \\ ${ }^{1}$ Graduate School of Engineering, University of Fukui, Fukui 910-8507, Japan \\ ${ }^{2}$ Faculty of Engineering, University of Fukui, Fukui 910-8507, Japan \\ ${ }^{3}$ Maintek Consultants, Yokohama 231-0024, Japan \\ Correspondence should be addressed to Tomomi Honda; honda@u-fukui.ac.jp
}

Received 30 April 2019; Accepted 25 October 2019; Published 7 February 2020

Academic Editor: Shyam Bahadur

Copyright $\odot 2020$ Tomohiko Kon et al. This is an open access article distributed under the Creative Commons Attribution License, which permits unrestricted use, distribution, and reproduction in any medium, provided the original work is properly cited.

Lubricating oils degrade into two main products: oxidation products and solid particles. Oxidation products, called varnish, of turbine oils for power generation have become a particularly serious problem in recent years. The first step in determining the potential to produce varnish is to determine the remaining life of the antioxidant in the oil, but even though turbine oil may have antioxidants of sufficient longevity, varnish problems still occur frequently. Accordingly, to prevent varnish, it is necessary to diagnose oil oxidation products. Thus, the authors have developed a diagnostic method using membrane patch color, but the relationship between membrane patch color and the remaining life of turbine oils has yet to be clarified. This paper investigates a new method for estimating the oxidative degradation of turbine oils that uses membrane patch color and the dry turbine oxidation stability test (dry TOST) based on oxidation products and the remaining life of the turbine oils. Sample oils were prepared and degraded by oxidation in the laboratory using a dry TOST apparatus, and the membrane patch color was measured using a colorimetric patch analyzer (CPA). The relationship between membrane patch color and the rotating pressure vessel oxidation test (RPVOT) residual rate was then investigated. The results show that the new estimation method using the CPA and dry TOST is able to monitor the decrease of the RPVOT residual rate from the early stages of oxidative deterioration.

\section{Introduction}

Lubricating oils degrade during operational use into two main products, namely, oil oxidation products and solid particles. In recent years, varnish-induced machine failures have become a serious problem with long-life turbine oils used in thermal power generation [1, 2]. Varnish is a thin deposit on metal surfaces that is caused by oxidation products in the lubricating oil; it can cause problems such as sticking and faulty operation of control valves, increased bearing temperature, bearing failure, blockage of oil filters, and impeded heat transfer. Many studies of varnish have been reported. Sasaki et al. [3] reported that oil oxidation products can be classified as either insoluble or soluble ones. Insoluble oxidation products are soluble in warm oil, but become insoluble when the oil is cooled. They also reported that soluble oil oxidation products have a molecular weight of 550-1900. Insoluble oil oxidation products with average molecular weight over 1900 are insoluble in oil even if it is warm. Johnson and Livingstone [4] proposed that the first step in determining the potential to produce varnish is to determine the remaining life of the antioxidant in the oil. This can be done for turbine oils either directly or indirectly. A direct antioxidant measurement method is the remaining useful life evaluation routine (RULER), whereas an indirect method is the rotating pressure vessel oxidation test (RPVOT) that is commonly used to evaluate the residual life of turbine oils. Both methods are very useful for evaluating the remaining life of turbine oil based on the remaining life of its antioxidant. Nevertheless, even though turbine oil may have antioxidants of sufficient longevity, varnish problems still occur frequently [5]. Therefore, to prevent varnish, it is important 
to evaluate not only the remaining life of turbine oil's antioxidants but also its oil oxidation products, both soluble and insoluble.

Thus, to diagnose oil oxidation products, the authors developed a new method for diagnosing the degradation of lubricating oils by focusing on the coloration of membrane patches that have been contaminated and a state-of-the-art colorimetric patch analyzer (CPA). The CPA can measure membrane patch color using both reflecting and transmitting light, whereas conventional colorimetric analyzers use only reflecting light. Oil oxidation products are collected on the surface of and inside the membrane filter, and the CPA uses transmitting light to measure the color information of oil oxidation products collected within the membrane filter in a way that conventional colorimetric analyzers cannot.

In previous studies [6,7], we reported a good relationship between membrane patch color and the oxidation products of turbine oils. As reported, membrane patch color is very useful and reliable for detecting oxidation products. However, the relationship between membrane patch color and the remaining life of turbine oils has yet to be clarified. Thus, to establish a new diagnostic method based on the presence of oxidation products using membrane patch color, it is necessary to investigate the relationship between membrane patch color and the remaining life of turbine oils. Yano et al. [8] proposed an estimation method based on the fact that the relationship between sludge resistance and the residual rate of the rotating bomb oxidation test (RBOT) can be estimated using the dry turbine oil stability test (dry TOST). For the present research, we used dry TOST to investigate the relationship between membrane patch color and RPVOT residual rate.

In this study, we prepared sample oils that were degraded by oxidation in the laboratory using a dry TOST apparatus. The remaining life of each sample oil was evaluated using RPVOT, and we investigated the relationship between membrane patch color and RPVOT residual rate. We also analyzed the manner in which changes in oxidation products corresponded to the RPVOT residual rate using Fouriertransform infrared spectroscopy (FT-IR). On the basis of the results of these studies, this paper discusses a new method for estimating the oxidative degradation of turbine oils that uses membrane patch color based on oxidation products and the remaining life of the turbine oils.

\section{Experimental Apparatus and Sample Oil}

2.1. Filtration Equipment. The filtration equipment comprised a dustproof cover, a filter support and a vacuum flask, a filter funnel, and a vacuum pump. Figure 1 shows a schematic of the filtering equipment. Figure 2 shows a magnified view of the surface of the membrane filter and its cross-sectional structure. The membrane filter was clamped between the filter support of the vacuum flask and the filter funnel. $25 \mathrm{~mL}$ of sample oil in the filter funnel was filtered with reduced pressure. We used a membrane filter with a pore size of $0.8 \mu \mathrm{m}$, a diameter of $25 \mathrm{~mm}$, and a thickness of $0.125 \mathrm{~mm}$. After filtration, the oil on the membrane patch was removed with petroleum ether, and the membrane filter

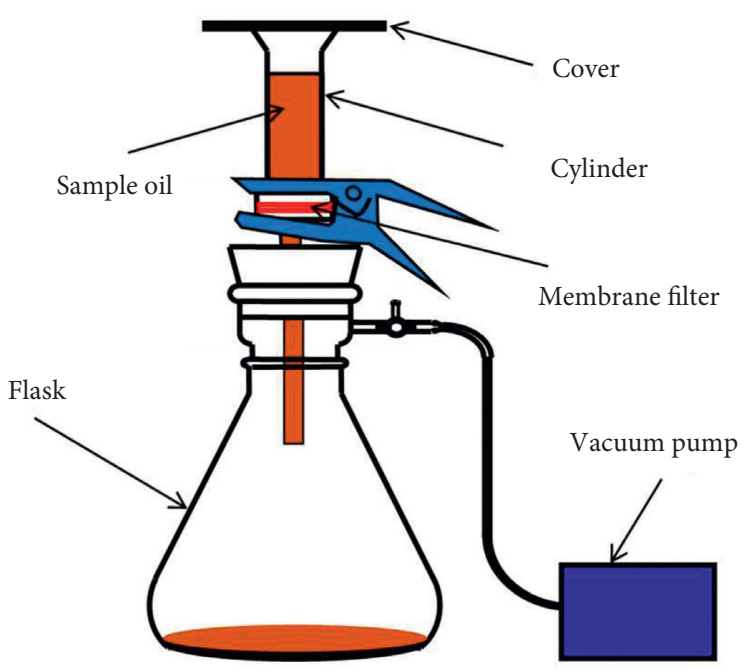

Figure 1: Filtering equipment.

that filtered the sample oil was dried; the dried membrane filter is called the "membrane patch."

2.2. Sample Oils and Filtration Procedure. Three samples of commercially available turbine oils with antioxidants were used. The oils were oxidized in stages using $120^{\circ} \mathrm{C}$ dry TOST, and the remaining life of these sample oils was evaluated using RPVOT. Table 1 lists the details of the sample oils. Sample oil A was oxidized in an air atmosphere, and sample oils $\mathrm{B}$ and $\mathrm{C}$ were oxidized in an oxygen atmosphere. The oxidation levels were changed with the dry TOST testing time. In addition, prior to filtering, the sample oils were heated to a temperature of $60^{\circ} \mathrm{C}-65^{\circ} \mathrm{C}$ for one day (while being stirred continuously) and then incubated and stored at room temperature away from UV light for three days in accordance with Section 8.1 of ASTM D7843.

\subsection{Colorimetric Patch Analyzer (CPA) and Color Parameters.} We measured the color parameters (maximum color difference and $\Delta E_{\mathrm{RGB}}$ ) of the membrane patch using a colorimetric patch analyzer (CPA). Figure 3 shows the CPA's measurement principle. The CPA projects white light alternately into the membrane patch from the top and bottom. Using the light that is reflected from the upper surface of the membrane filter, the CPA measures the color information of contaminants captured on the surface of the membrane filter. Using the light that is transmitted through the membrane filter from the bottom, the CPA measures the color information of contaminants captured on the surface and the inside of the membrane filter. As mentioned in Section 1, oil oxidation products collect both inside the membrane filter and on its surface. For this method, we used the transmitting light to obtain all required information about the oxidation products captured by the membrane filter.

The color parameters (i.e., the maximum color difference and $\Delta E_{\mathrm{RGB}}$ ) are calculated from RGB values that are indicated in 256 levels. For example, white has R, G, and B all at 


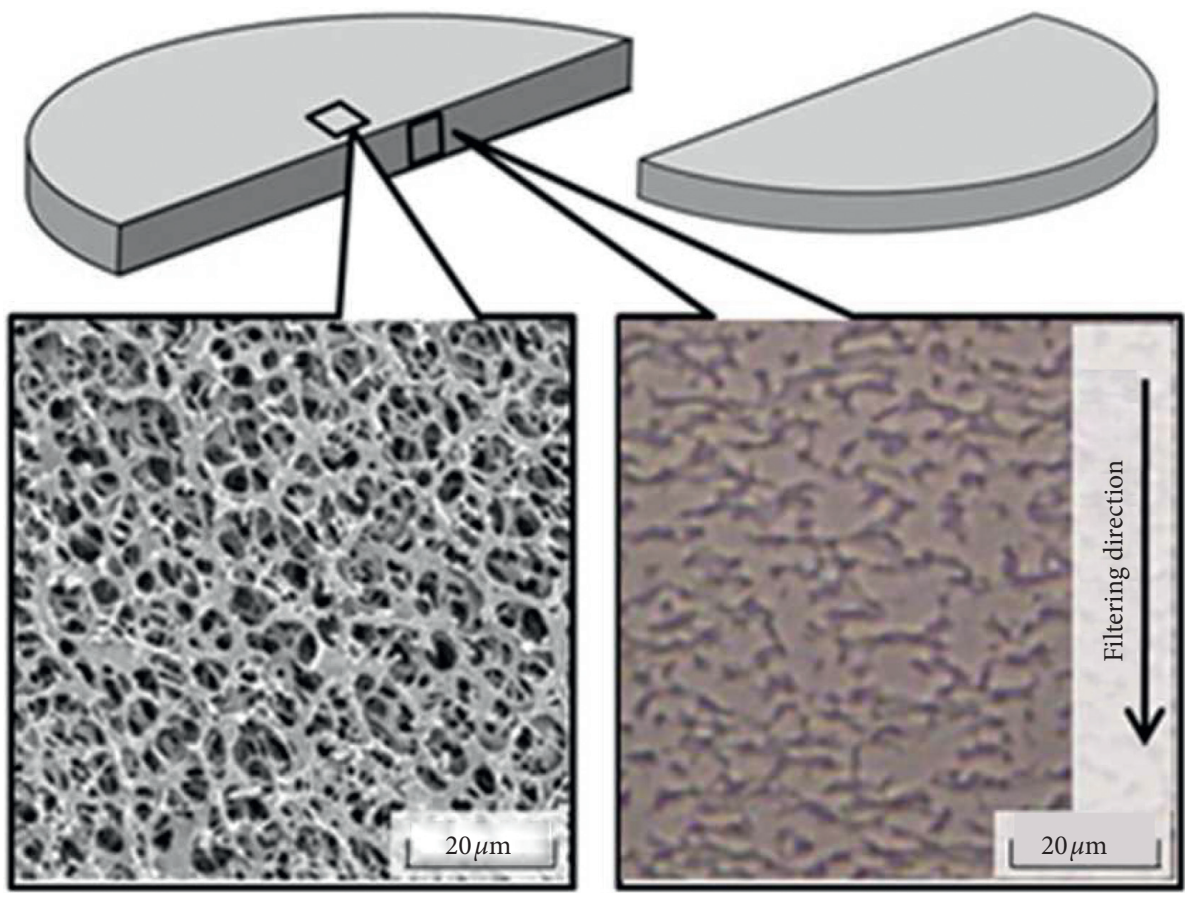

(a)

(b)

Figure 2: Magnified images of the membrane filter. (a) Surface. (b) Cross section.

TABLe 1: Properties of the solvents.

\begin{tabular}{lccc}
\hline & Dry TOST testing time $(\mathrm{h})$ & RPVOT value $(\mathrm{min})$ & RPVOT residual rate $(\%)$ \\
\hline & 0 & 1250 & 100 \\
Sample oil A & 1003 & 765 & 61.2 \\
& 1884 & 530 & 42.4 \\
& 2500 & 420 & 33.6 \\
& 0 & 1648 & 100 \\
Sample oil B & 336 & 1140 & 69.2 \\
& 456 & 900 & 54.6 \\
& 504 & 580 & 35.2 \\
& 558 & 520 & 31.6 \\
Sample oil C & 600 & 350 & 21.2 \\
& 678 & 300 & 18.2 \\
& 0 & 2605 & 100 \\
& 336 & 2485 & 95.4 \\
& 1008 & 1690 & 64.9 \\
& 1395 & 1020 & 39.2 \\
\end{tabular}

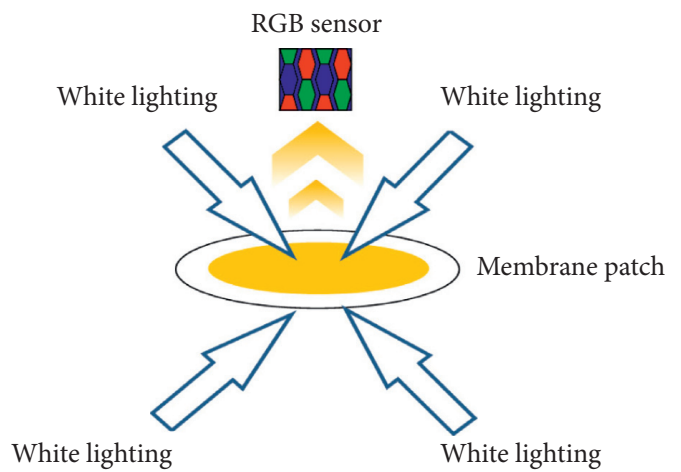

FIgURE 3: Measurement principle of the CPA. 
TABLE 2: Membrane patch images and RGB values.

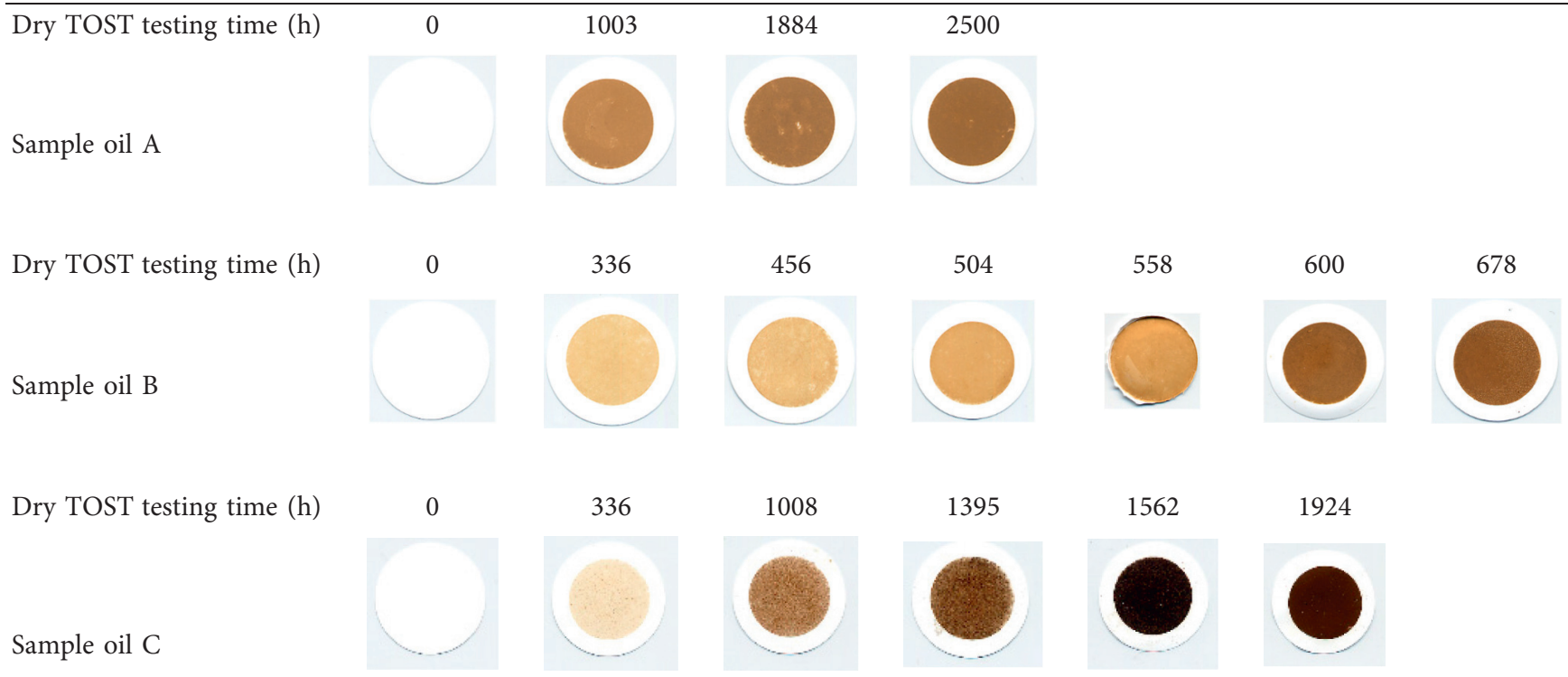

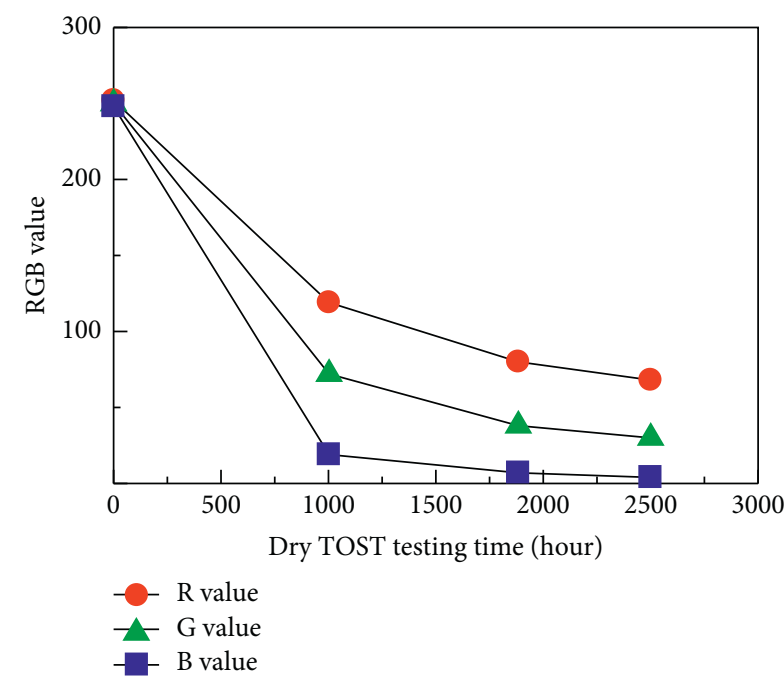

(a)

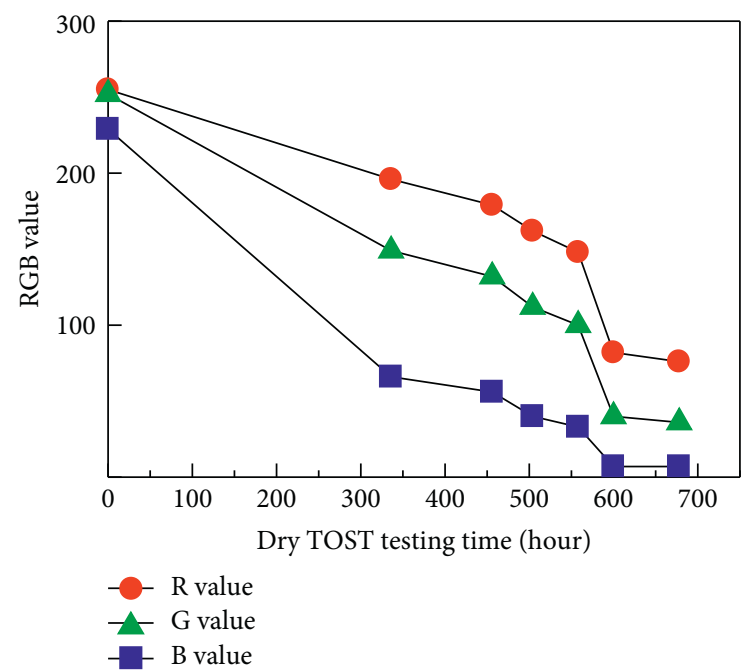

(b)

Figure 4: Continued. 


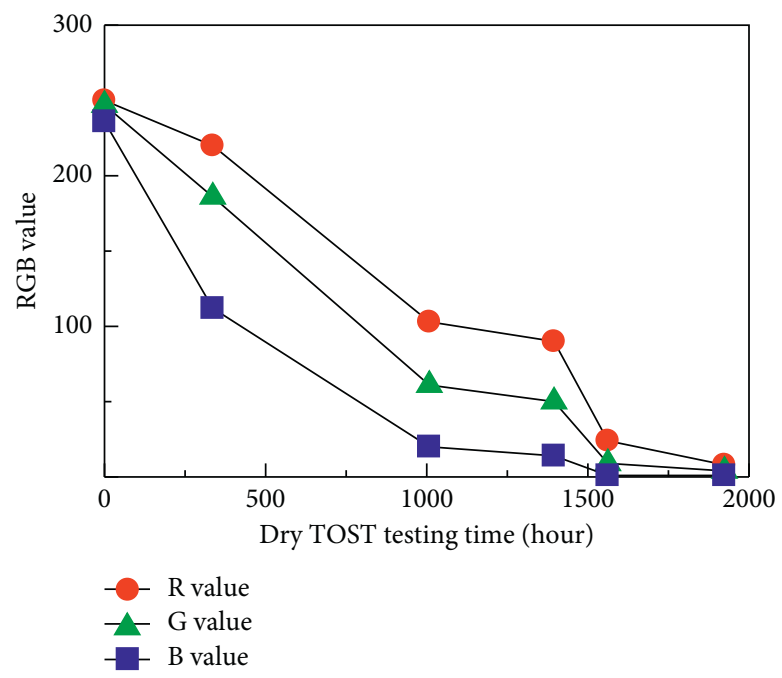

(c)

FIgURE 4: Relationship between dry TOST testing time and RGB values. (a) Sample oil A. (b) Sample oil B. (c) Sample oil C.

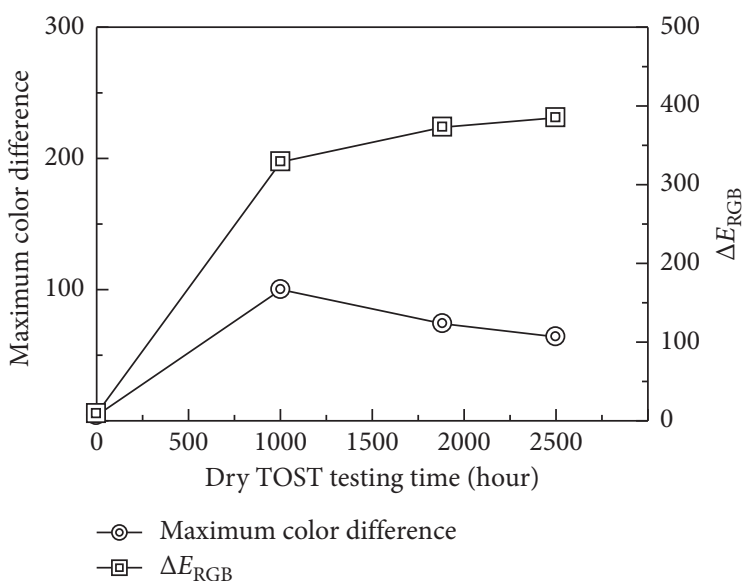

(a)

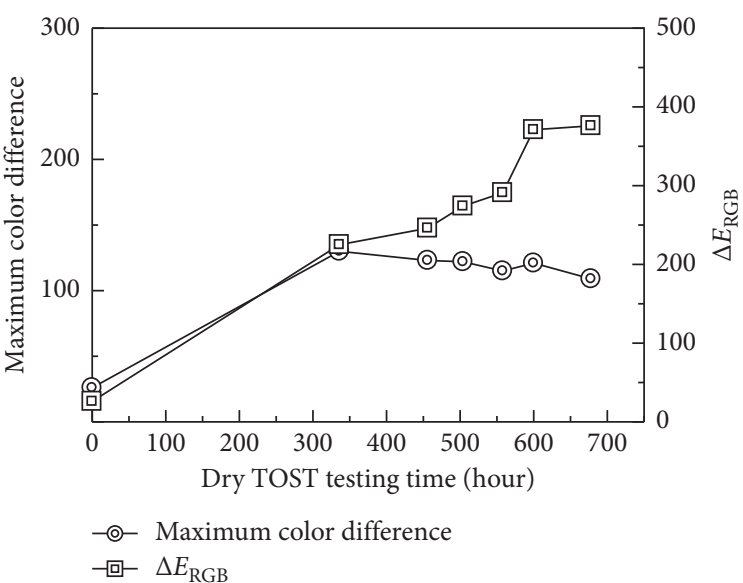

(b)

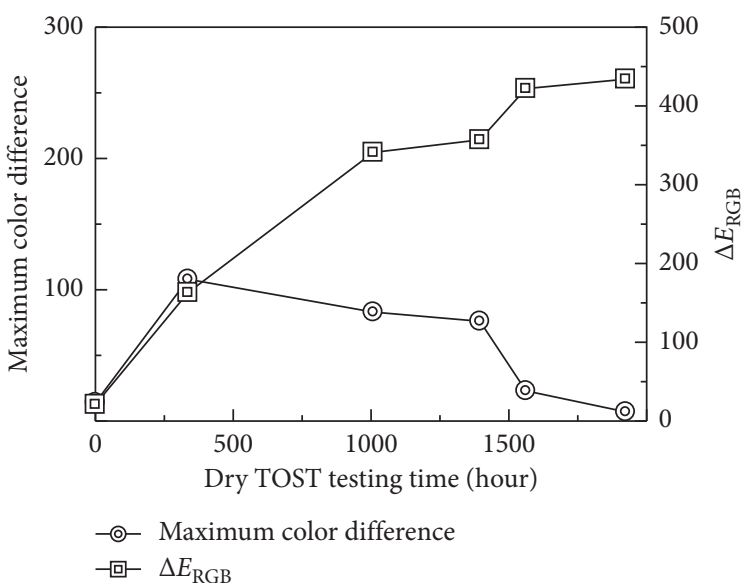

(c)

FIGURE 5: Relationship between dry TOST testing time, the maximum color difference, and $\Delta E_{\text {RGB }}$. (a) Sample oil A. (b) Sample oil B. (c) Sample oil C. 


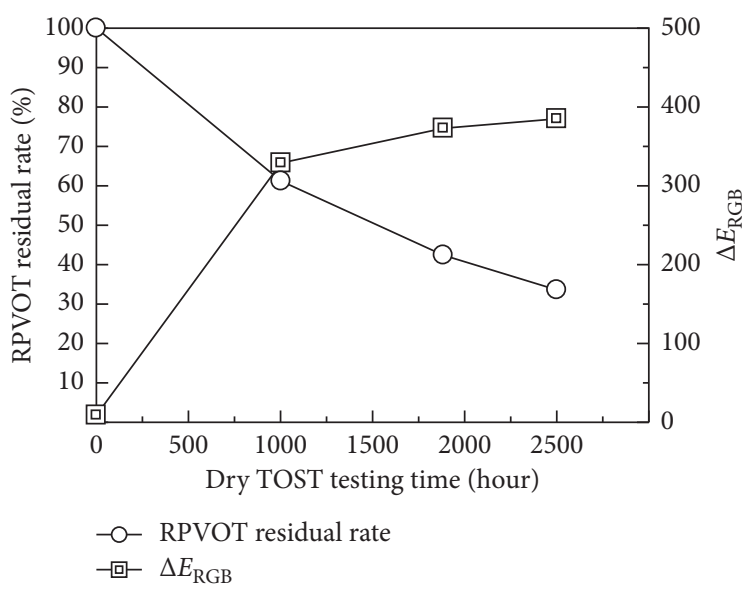

(a)

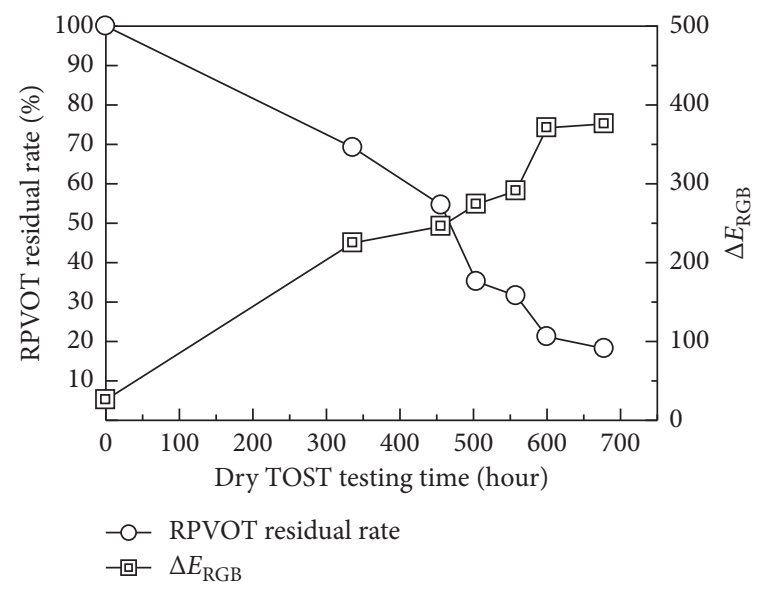

(b)

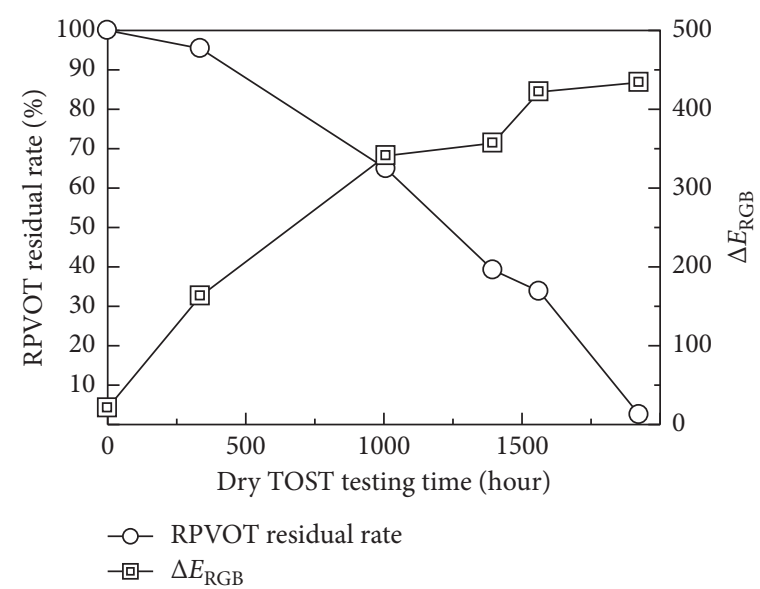

(c)

FIGURE 6: Relationship between Dry TOST testing time, PVOT residual rate and $\Delta E_{\text {RGB. }}$ (a) Sample oil A. (b) Sample oil B. (c) Sample oil C.

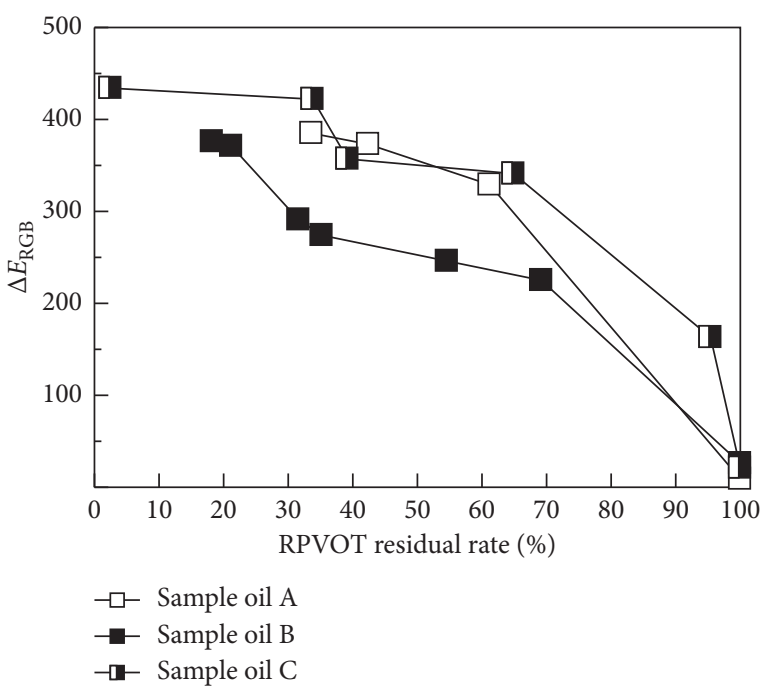

FIGURE 7: Relationship between RPVOT residual rate and $\Delta E_{\mathrm{RGB}}$.

level 256, and black has R, G, and B all at level 0 . The maximum color difference is defined as the maximum difference between the two values of R, G, and B; it has a close relationship with the degradation factors of lubricating oils [6]. The quantity $\Delta E_{\mathrm{RGB}}$ is defined as the color distance between white and the membrane patch color as obtained using (1); $\Delta E_{\mathrm{RGB}}$ is used to measure the degradation level of the lubricating oils. The RPVOT residual rate is the RPVOT value of the used oil divided by the RPVOT value of new oil, expressed in percentage; it is obtained using (2). The value RPVOT $(t)$ is the RPVOT value after $t$ hours of degradation and indicates how much residual life remains for the turbine oil by comparing it with new oil. In this study, the RPVOT residual rate was used as an index to evaluate the residual life of the turbine oils.

$$
\begin{gathered}
\Delta E_{\mathrm{RGB}}=\left\{(255-\mathrm{R})^{2}+(255-\mathrm{G})^{2}+(255-\mathrm{B})^{2}\right\}^{0.5}, \\
\mathrm{RPVOT} \text { residual rate }=\frac{\operatorname{RPVOT}(t)}{\operatorname{RPVOT}(\text { new })} \times 100 .
\end{gathered}
$$

\section{Results and Discussion}

3.1. Relationships between Dry TOST Testing Time and RGB Values and Membrane Patch Color. Table 2 shows the images of the membrane patches, and Figure 4 shows the 


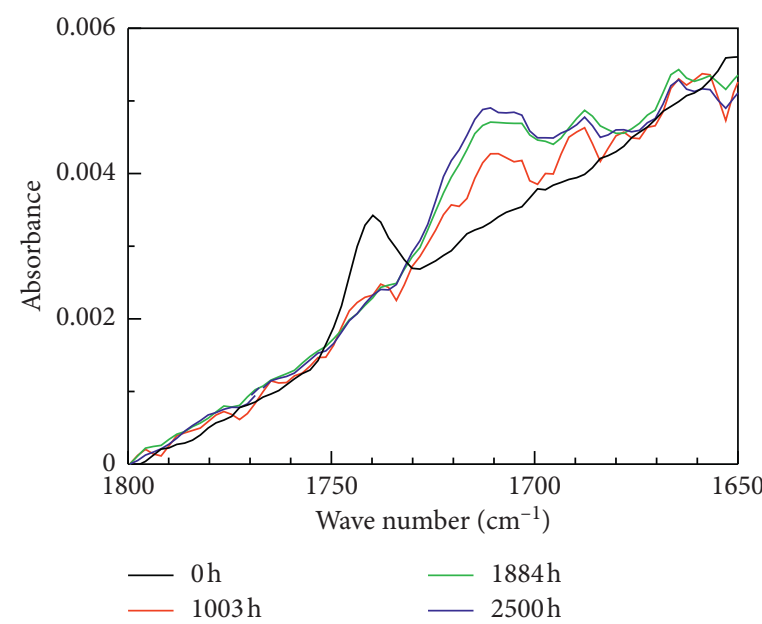

(a)

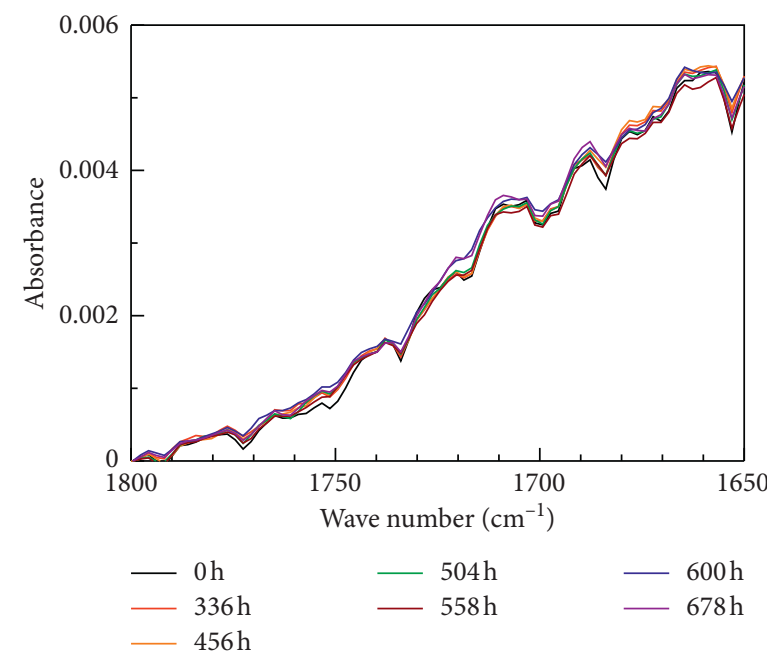

(b)

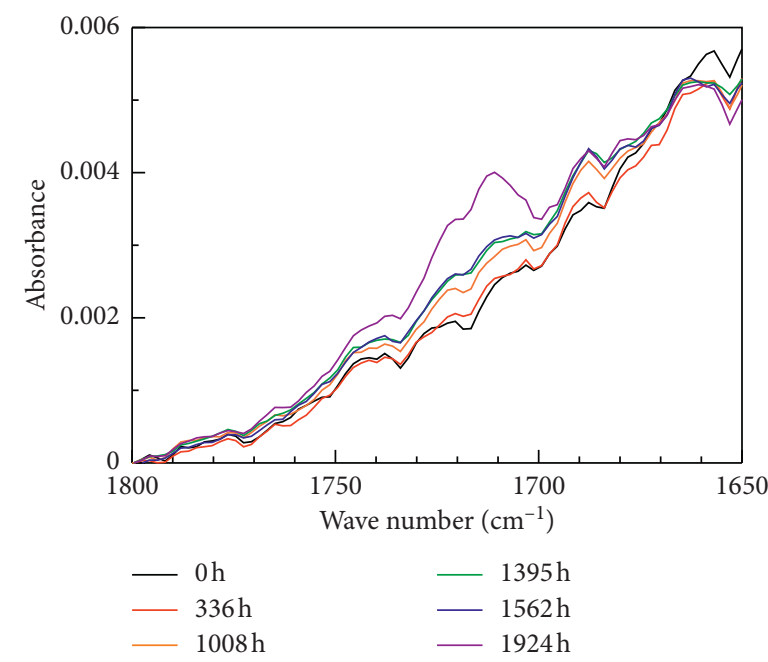

(c)

FIGURE 8: IR spectra of sample oils from 1800 to $1650 \mathrm{~cm}^{-1}$. (a) Sample oil A. (b) Sample oil B. (c) Sample oil C.

relationship between the dry TOST testing time and the RGB values measured using transmitting light. When the membrane patch color is measured using transmitting light, there is no way to display the measured color directly. Instead, Table 2 shows the appearance of the membrane patches in reflecting light. For sample oils A and B, the membrane patch turned dark brown as the dry TOST testing time increased. Similarly, the membrane patch for sample oil $\mathrm{C}$ darkened as the dry TOST testing time increased. The RGB values of the sample oils decreased with the dry TOST testing time. Figure 5 shows the relationships between dry TOST testing time, the maximum color difference and $\Delta E_{\mathrm{RGB}}$. $\Delta E_{\mathrm{RGB}}$ increased with the dry TOST testing time for each sample oil; however, its greatest increase occurred in sample oil B, i.e., between 558 and $600 \mathrm{~h}$.

3.2. Relationship between RPVOT Residual Rate and Membrane Patch Color. Figure 6 shows the relationships between dry TOST testing time, RPVOT residual rate, and $\Delta E_{\mathrm{RGB}}$.
The RPVOT residual rate decreased and $\Delta E_{\mathrm{RGB}}$ increased with increasing dry TOST testing time for each sample oil. Moreover, the decreasing tendency of the RPVOT residual rate and the increasing tendency of $\Delta E_{\mathrm{RGB}}$ differed among the sample oils.

Figure 7 shows the relationship between RPVOT residual rate and $\Delta E_{\mathrm{RGB}} . \Delta E_{\mathrm{RGB}}$ increased as the RPVOT residual rate decreased, which indicates there is a correlation between RPVOT residual rate and $\Delta E_{\mathrm{RGB}}$. ASTM D4378 defines the RPVOT limit as $25 \%$.

In sample oil $\mathrm{B}, \Delta E_{\mathrm{RGB}}$ greatly increased when the RPVOT residual rate fell below $25 \%$. However, $\Delta E_{\mathrm{RGB}}$ for sample oil C greatly increased when the RPVOT residual rate was between $40 \%$ and $34 \%$. It seems that different turbine oils have different degradation tendencies, making it necessary to specify the RPVOT limit for each turbine oil. In our tests, if $\Delta E_{\mathrm{RGB}}$ reached approximately 350 , the sample oil reached its RPVOT limit. Thus, we conclude that the RPVOT residual rate of actual turbine oils can be estimated easily using $\Delta E_{\mathrm{RGB}}$ if a calibration curve is prepared using 
dry TOST, as shown in Figure 7. Therefore, if turbine oils are managed using this calibration curve, good conditions can be maintained in relation to oxidation products and remaining life.

3.3. FT-IR Analysis of Sample Oils. Figure 8 shows the IR spectra expanded from 1800 to $1650 \mathrm{~cm}^{-1}$ to identify the oxidation product peaks. These were adjusted such that an absorbance of $1800 \mathrm{~cm}^{-1}$ corresponds to zero. The level of absorbance at approximately $1710 \mathrm{~cm}^{-1}$, which is the absorption band peak of the carbonyl group [9], increased with dry TOST testing time for sample oils A and C. However, no such relationship was found for sample oil B which we knew to be oxidized because the RPVOT residual rate decreased with the dry TOST testing time. However, the peak for the oxidation products did not increase with dry TOST testing time. As a result, sample oil B could not be diagnosed as having increased oxidation products. Therefore, we conclude that degradation of oil of the same type as sample oil B, which is difficult to diagnose using FT-IR analysis, can be estimated easily using the proposed method based on colorimetric analysis.

\section{Conclusions}

In the present study, it was found that the RPVOT residual rate and the $\Delta E_{\mathrm{RGB}}$ data show similar tendencies, and our proposed estimation method could be used to estimate the remaining life of turbine oils.

\section{Data Availability}

The data used to support the findings of this study are available from the corresponding author upon request.

\section{Conflicts of Interest}

The authors declare that there are no conflicts of interest regarding the publication of this paper.

\section{Acknowledgments}

This work was supported by KAKENHI (grant number 25420086) of the Japan Society for the Probehavior of Science (JSPS), Japan.

\section{References}

[1] L. Day, "Solving varnish problems in gas turbine lubricants," Tribology and Lubrication Technology, vol. 64, no. 1, pp. 32-38, 2008.

[2] G. Livingstone and D. Oakton, "The emerging problem of lubricant varnish," Maintenance \& Asset Management Journal, vol. 25, no. 2, pp. 38-42, 2010.

[3] A. Sasaki, H. Aoyama, T. Honda, Y. Iwai, and C. K. Yong, "A study of the colors of contamination in used oils," Tribology Transactions, vol. 57, no. 1, pp. 1-10, 2014.

[4] M. Johnson and G. Livingstone, "Identifying varnish and oxidation precursors in lubricant sumps," Tribology and Lubrication Technology, vol. 67, no. 4, pp. 2-7, 2011.
[5] A. Sasaki, S. Uchiyama, and M. Kawasaki, "Varnish formation in the gas turbine oil systems," Journal of ASTM International, vol. 5, no. 7, pp. 103-114, 2008.

[6] T. Yamaguchi, S. Kawamura, T. Honda, M. Ueda, Y. Iwai, and A. Sasaki, "Investigation of oil contamination by colorimetric analysis," Lubrication Engineering, vol. 58, no. 1, pp. 12-17, 2002.

[7] T. Honda and A. Sasaki, "Development of a turbine oil contamination diagnosis method using colorimetric analysis of membrane patches," Journal Bulletin of the JSME, vol. 12, no. 4, pp. 18-00277, 2018.

[8] A. Yano, S. Watanabe, Y. Miyazaki, M. Tsuchiya, and Y. Yamamoto, "Study on sludge formation during the oxidation process of turbine oils," Tribology Transactions, vol. 47, no. 1, pp. 111-122, 2004.

[9] M. S. Robert and X. W. Francis, Spectrometric Identification of Organic Compounds, John Wiley \& Sons, Hoboken, NJ, USA, 6th edition, 1998. 